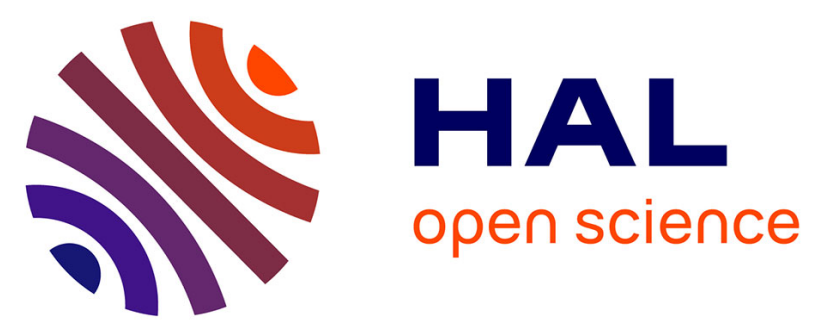

\title{
Simultaneous Localization and Mapping in Millimeter Wave Networks with Angle Measurements
}

\author{
Remun Koirala, Benoit Denis, Bernard Uguen, Davide Dardari, Henk
}

Wymeersch

\section{- To cite this version:}

Remun Koirala, Benoit Denis, Bernard Uguen, Davide Dardari, Henk Wymeersch. Simultaneous Localization and Mapping in Millimeter Wave Networks with Angle Measurements. 2020 IEEE International Conference on Communications Workshops (ICC Workshops), Jun 2020, Dublin, Ireland. pp.1-6, 10.1109/ICCWorkshops49005.2020.9145046 . cea-02949954

\section{HAL Id: cea-02949954 https://hal-cea.archives-ouvertes.fr/cea-02949954}

Submitted on 26 Sep 2020

HAL is a multi-disciplinary open access archive for the deposit and dissemination of scientific research documents, whether they are published or not. The documents may come from teaching and research institutions in France or abroad, or from public or private research centers.
L'archive ouverte pluridisciplinaire HAL, est destinée au dépôt et à la diffusion de documents scientifiques de niveau recherche, publiés ou non, émanant des établissements d'enseignement et de recherche français ou étrangers, des laboratoires publics ou privés. 


\title{
Simultaneous Localization and Mapping in Millimeter Wave Networks with Angle Measurements
}

\author{
Remun Koirala*†‡, Benoît Denis* $^{*}$, Bernard Uguen ${ }^{\ddagger}$, Davide Dardari ${ }^{\dagger}$ and Henk Wymeersch ${ }^{\S}$ \\ ${ }^{*}$ CEA-Leti Minatec Campus, 17 rue des Martyrs, 38054 Grenoble Cedex 09, France \\ $\dagger$ DEI-CNIT, University of Bologna, via dell’Università 52, I-47521 Cesena (FC), Italy \\ $\ddagger$ University of Rennes 1-IETR (CNRS UMR 6164), Av. du General Leclerc, 35042, Rennes, France \\ $\S$ Department of Electrical Engineering, Chalmers University of Technology, Gothenburg, Sweden
}

\begin{abstract}
In this paper we propose a belief propagation (BP) based simultaneous localization and mapping (SLAM) approach suitable for millimeter wave (mm-Wave) networks. This approach leverages angle of arrival (AoA) and angle of departure (AoD) information with respect to multiple scatterers. Considering measurements from multiple base stations (BSs) and scatterers, seen as multiple sources, we solve out the data association problem from a centralized BP perspective, while jointly estimating the positions of both the mobile and scatterers. Simulations show that the proposed approach outperforms conventional distributed BSwise BP methods in terms of estimation accuracy.
\end{abstract} tion

Index Terms-SLAM, loopy belief propagation, data associa-

\section{INTRODUCTION}

With the increasing traffic congestion in the microwave frequency band, the interest has been burgeoning towards exploring the yet underused large bandwidths available in the millimeter band spanning from $30 \mathrm{GHz}$ to $300 \mathrm{GHz}$ [1]. However, at such high frequency, the transmission suffers from high path loss, further signal attenuation caused by environmental factors (e.g., rain, fog) and even more importantly, high susceptibility to blockages [2], [3]. In order to combat such effects, millimeter wave (mm-Wave) systems utilize a large number of antennas to concentrate the signal power in a particular direction with beamforming [4].

The advantages of exploiting a large bandwidth and a large number of antennas at both transmitter and receiver however are pertinent not only to mm-Wave communications but also to localization [5]. These features allow for high temporal and angular resolution, enabling accurate delay and angle (angle of departure (AoD) and angle of arrival (AoA)) estimation. In addition, the mm-Wave propagation channel is sparse by nature meaning that there are only few multipath components. Consequently, in mm-Wave system, it is possible to estimate the delays, AoAs and AoDs of the main multipath components [6]. Thus, one can leverage the location-dependent information conveyed by each component to aid the localization process [7]. Beyond, knowing the positions of the scatterers generating these multipath contributions, in addition to localizing the user, offers a unique opportunity to map the physical environment.

With regards to tracking the location dependent variables of both line-of-sight (LOS) and non line of sight (NLOS) components, the authors in [8] and [9] present an estimator, exploiting the mm-Wave channel sparsity, relying on simultaneous orthogonal matching pursuit (SOMP) and support detection (SD) algorithms. Similarly, the authors in [10] and [11] present an algorithm for simultaneous localization and mapping (SLAM) based on the multiple location estimates of the user and the scatterers at different time instances. In [12], the authors present a message passing based solution for estimating the position and orientation of the user and position of the scattering point in both presence and absence of LOS components. The authors show that even in the absence of LOS path, the position of the scatterers can be reliably estimated. Likewise, in [13], the authors provide a belief propagation (BP) based approach to track features in the environment in a dynamic vehicular scenario. The authors in [14] propose a BP based algorithm to solve the data association problem (associating measurements to the correct source) and present a low complexity implementation of the corresponding solver. Common to all methods that rely on time-based measurements is the need for tight synchronization [12], [15]. Localization using only angular information avoids this bottleneck. Cooperative localization using only AoA information was proposed in [16]. Similarly, in [17], the authors investigate indoor localization with only AoA measurements.

In this paper, we present a BP based SLAM approach in order to jointly localize the user and the scatterers relying only on angle measurements, without a-priori knowledge of the positions of either the user or the scatterers. In the process, we also solve the data association problem concerned with the measurements and their corresponding sources ${ }^{1}$. The processing is done in a centralized way without needing to send the measurements to each base station (BS), in contrast

\footnotetext{
${ }^{1}$ The term "source" can refer to either the user or the scatterers.
} 


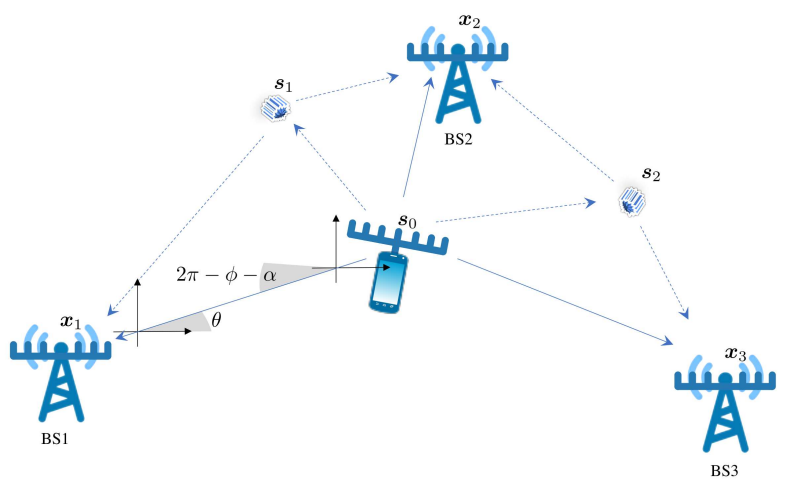

Fig. 1. Example system model with 3 BSs positioned at $\boldsymbol{x}_{1}, \boldsymbol{x}_{2}$ and $\boldsymbol{x}_{3}$ and 1 user positioned at $\boldsymbol{s}_{0}$ with orientation $\alpha$ along with two scatterers at $\boldsymbol{s}_{1}$ and $s_{2}$ and the AoD and AoA of the LOS paths with respect to BS1.

to more conventional distributed BP approaches [13]. One major motivation for adopting a centralized approach in the very context lies in its lower communication overhead. The distributed method indeed requires sharing of particle clouds among the different BSs, while our method just requires sharing of raw measurements and estimates.

\section{SySTEM MODEL}

Consider a 2 dimensional (2D) scenario with $N$ BSs and a single user as illustrated in Fig. 1. The positions of the BSs are assumed to be known, located at $\boldsymbol{x}_{1}, \boldsymbol{x}_{2}, \cdots, \boldsymbol{x}_{N}$ in a common coordinate system. Likewise, the user is assumed to be positioned at $s_{0}$ with a known orientation ${ }^{2} \alpha$. In the scenario, we also assume $L$ scattering points (and hence $L+1$ total possible paths between a BS and the mobile user), each with positions $s_{1}, s_{2}, \cdots, s_{L}$. Both the positions of the user and the scatterers are unknown. Contrarily to [7] (where range measurements are also considered), here we assume that each BS $i$ can measure the AoD and AoA pair of $\hat{L}_{i}$ different paths ${ }^{3}$. We also assume that $L$ is known, there are no false alarms ${ }^{4}$, i.e. $\hat{L}_{i} \leq L+1$, and the measurements can be in any order with respect to the $L+1$ total paths.

$$
\mathcal{Z}^{(i)}=\left[\boldsymbol{z}_{1}^{(i)}, \cdots, \boldsymbol{z}_{l}^{(i)}, \cdots, \boldsymbol{z}_{\hat{L}_{i}}^{(i)}\right]
$$

where $l \in\left\{1,2, \cdots, \hat{L}_{i}\right\}$ denotes the measurement index of $i$ th BS corresponding to a set of estimates of $\operatorname{AoD}(\hat{\theta})$ and AoA $(\hat{\phi})$. We also assume that we have the measurement covariance matrix $\left(\Sigma_{l}^{(i)}\right)$ corresponding to the measurements of AoD and AoA between the $i$-th $\mathrm{BS}$ and the $l$-th scatterer.

\footnotetext{
${ }^{2}$ For simplicity, we consider that the orientation of the user is known. The absolute heading can be extracted from some orientation estimators, for instance, inertial measurement unit (IMU) and magnetometer in the mobile phone [10].

${ }^{3}$ Note that it is not necessary that a BS has AoD and AoA measurements corresponding to every path in the system. Hence, we use different notations for the total number of paths $(L)$ and the total number of paths measured by the $i$-th BS $\left(\hat{L}_{i}\right)$.

${ }^{4}$ This consideration excludes the possibility of any measurements not associated with the user, $N$ BSs and $L$ scatterers. For the problem formulation including the possibility of false alarm, see [14].
}

We consider that all the measurements $\boldsymbol{z}_{l}^{(i)}$ are independent. Mathematically,

$$
\boldsymbol{z}_{l}^{(i)}=\left[\hat{\theta}_{l}^{(i)}, \hat{\phi}_{l}^{(i)}\right] \sim \mathcal{N}\left(\left[\theta_{l}^{(i)}, \phi_{l}^{(i)}\right]^{T}, \boldsymbol{\Sigma}_{l}^{(i)}\right) .
$$

The measurements for all the $N$ BSs can be grouped together as $\boldsymbol{Z}=\left[\mathcal{Z}^{(1)}, \cdots, \mathcal{Z}^{(N)}\right]$.

The main objective of this paper is to determine the positions of the user and that of all the scattering points from angle measurements, and thus the main task is to calculate the posterior distribution $p\left(s_{k} \mid \boldsymbol{Z}\right)$, where $k \in\{0,1, \cdots, L\}$. However, since the order of the measurements at each BS is random, in the process of calculating the posterior distribution, we also need to solve the data association problem, where we associate each measurement with the corresponding path.

\section{FACTOR GRAPH FORMULATION}

\section{A. Data Association Auxiliary Variables}

For the purpose of data association, following a similar approach to [13], we introduce the sets $\mathcal{M}=\otimes_{i=1}^{N}\left\{1, \cdots, \hat{L}_{i}\right\}$ with its cardinality $P=\prod_{i=1}^{N} \hat{L}_{i}, \boldsymbol{a}_{k} \in \mathcal{M}$ and $b_{\boldsymbol{m}} \in$ $\{0,1, \cdots, L\}, \forall \boldsymbol{m} \in \mathcal{M}$. Each element of the set $\mathcal{M}$, referred to in this paper as a measurement vector, is a vector of length $N$ containing all the possible permutations of measurement indices; $\boldsymbol{a}_{k}$ indicates which measurement vector corresponds to the source $k$. Reciprocally, $b_{m}$ indicates which source corresponds to a measurement vector. Both $\boldsymbol{a}$ and $b$ can be mapped one-to-one, meaning that the knowledge of either of the variables is sufficient to know the other. Mathematically, this relation is expressed as

$$
\psi\left(\boldsymbol{a}_{k}, b_{\boldsymbol{m}}\right)= \begin{cases}0 & \boldsymbol{a}_{k}=\boldsymbol{m}, b_{\boldsymbol{m}} \neq k \text { or } \boldsymbol{a}_{k} \neq \boldsymbol{m}, b_{\boldsymbol{m}}=k, \\ 1 & \text { otherwise. }\end{cases}
$$

Contrary to [13], where the data association is performed in a distributed way at each BS, we assume that the association in our case is done in a centralized way and hence, unlike in [13], the association variable $\boldsymbol{a}$ in our work is not scalar.

\section{B. Factor Graph of Joint Distribution}

Our objective is to find the marginal probability of $s_{k}$ while also sorting out the data association problem. We have a posterior distribution formulation as follows:

$$
p\left(\boldsymbol{s}_{0: L}, \boldsymbol{a}, \boldsymbol{b} \mid \boldsymbol{Z}\right) \propto p\left(\boldsymbol{Z} \mid \boldsymbol{s}_{0: L}, \boldsymbol{a}, \boldsymbol{b}\right) \Psi(\boldsymbol{a}, \boldsymbol{b}) \prod_{k=0}^{L} p\left(\boldsymbol{s}_{k}\right),
$$

where, $p\left(\boldsymbol{s}_{k}\right)$ is the prior distribution on the position of the sources, $\boldsymbol{a}$ and $\boldsymbol{b}$ contain all the association variables $\boldsymbol{a}_{k}$ and $b_{\boldsymbol{m}}$, and $\psi(\boldsymbol{a}, \boldsymbol{b})$ can be formulated similarly to [13], [14] from (3):

$$
\Psi(\boldsymbol{a}, \boldsymbol{b})=\prod_{k=0}^{L} \prod_{\boldsymbol{m} \in \mathcal{M}} \psi\left(\boldsymbol{a}_{k}, b_{\boldsymbol{m}}\right) .
$$

The measurement likelihood $p\left(\boldsymbol{Z} \mid s_{0: L}, \boldsymbol{a}, \boldsymbol{b}\right)$ can be formulated as:

$$
p\left(\boldsymbol{Z} \mid \boldsymbol{s}_{0: L}, \boldsymbol{a}, \boldsymbol{b}\right)=\prod_{i=1}^{N} p\left(\mathcal{Z}^{(i)} \mid \boldsymbol{s}_{0: L}, \boldsymbol{a}, \boldsymbol{b}\right),
$$




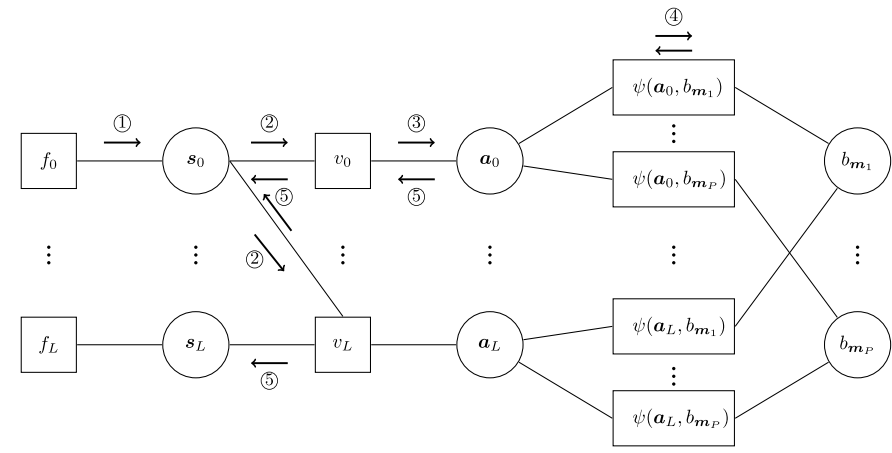

Fig. 2. Factor graph representation of the posterior distribution in equation (4). In the graph, we have introduced short form notation $f_{k}$ to represent $p\left(s_{k}\right), v_{k}$ to represent $v\left(a_{k}, s_{0}, s_{k}\right)$ and $\boldsymbol{m}_{p}, p=1, \cdots, P$ represents the $p$-th element of the set $\mathcal{M}$. The factor and variable nodes are represented inside squares and circles respectively.

$$
\begin{aligned}
& \propto \prod_{k=0}^{L} \prod_{i=1}^{N} p\left(\boldsymbol{z}_{\left[\boldsymbol{a}_{k}\right]_{i}}^{i} \mid \boldsymbol{s}_{0}, \boldsymbol{s}_{k}\right), \\
& \propto \prod_{k=0}^{L} v\left(\boldsymbol{a}_{k}, \boldsymbol{s}_{0}, \boldsymbol{s}_{k}\right) .
\end{aligned}
$$

where, the expression $v\left(\boldsymbol{a}_{k}, \boldsymbol{s}_{0}, \boldsymbol{s}_{k}\right)=\prod_{i=1}^{N} p\left(\boldsymbol{z}_{\left[\boldsymbol{a}_{k}\right]_{i}}^{i} \mid \boldsymbol{s}_{0}, \boldsymbol{s}_{k}\right)$ represents the joint AoD-AoA distribution considering all the BSs and $p\left(\boldsymbol{z}_{\left[\boldsymbol{a}_{k}\right]_{i}}^{i} \mid \boldsymbol{s}_{0}, \boldsymbol{s}_{k}\right)$ is Gaussian distributed, as per (2): $p\left(\boldsymbol{z}_{\left[\boldsymbol{a}_{k}\right]_{i}}^{i} \mid \boldsymbol{s}_{0}, \boldsymbol{s}_{k}\right) \propto \exp \left(-\left\|\left[\hat{\theta}_{\left[\boldsymbol{a}_{k}\right]_{i}}^{(i)}, \hat{\phi}_{\left[\boldsymbol{a}_{k}\right]_{i}}^{(i)}\right]-\boldsymbol{h}\left(\boldsymbol{s}_{0}, \boldsymbol{s}_{k}\right)\right\|_{\Sigma_{\left[\boldsymbol{a}_{k}\right]_{i}}^{(i)}}\right)$,

where $\|\boldsymbol{x}\|_{\boldsymbol{A}}=\boldsymbol{x}^{T} \boldsymbol{A} \boldsymbol{x}$ and $\boldsymbol{h}\left(\boldsymbol{s}_{0}, \boldsymbol{s}_{k}\right)$ is a nonlinear function transforming the location of the user $s_{0}$ in the case of direct path and both the user $s_{0}$ and the scatterer $s_{l}$ in the case of non-direct path to the corresponding AoD and AoA variables.

Note that from equation (6b), one does not have the dependence on $\boldsymbol{b}$, as the equation (6a) is conditioned on the knowledge of both $\boldsymbol{a}$ and $\boldsymbol{b}$ and since $\boldsymbol{a}$ and $\boldsymbol{b}$ are injective-only, knowing one is enough to recover the other. The corresponding factor graph of the posterior distribution is given in Fig. 2.

\section{Message Passing Via BP}

\section{A. BP for Marginalization}

We find the marginal $p\left(s_{k} \mid \boldsymbol{Z}\right)$ using the BP message passing algorithm on the factor graph [18], starting from the root nodes $p\left(\boldsymbol{s}_{k}\right)$ to the leaf node ${ }^{5}$. BP proceeds by passing messages between variables and factors defined by $\mu_{v \rightarrow f}(v)$ and $\mu_{f \rightarrow v}(v)$, respectively, where $v$ is a variable and $f$ is a factor. The marginal $p\left(\boldsymbol{s}_{k} \mid \boldsymbol{Z}\right)$ can then be found as

$$
p\left(\boldsymbol{s}_{k} \mid \boldsymbol{Z}\right) \propto \mu_{\boldsymbol{s}_{k} \rightarrow f}\left(\boldsymbol{s}_{k}\right) \times \mu_{f \rightarrow \boldsymbol{s}_{k}}\left(\boldsymbol{s}_{k}\right),
$$

for any connected factor $f$.

\footnotetext{
${ }^{5}$ Note that the factor graph in Fig. 2 consists of loops in between the variables $\boldsymbol{a}$ and $\boldsymbol{b}$. In such loopy cases, BP can still be used by passing the messages until the latter converge. Even though such convergence can not be guaranteed, it has been shown that this kind of method often arrives at a reasonable estimate [19].
}

\section{B. Message Passing Schedule}

We now show the message passing steps from the root nodes $f_{k}$ through the nodes back to the leaf node $f_{k}$. The root node $f_{k}$ or $p\left(s_{k}\right)$ contains the a-priori distribution of the position of $s_{k}$. In the following, we consider the indices $1 \leq k^{\prime} \leq L$ and $0 \leq k \leq L$.

(1) In the first step, we pass the a-priori distributions to the corresponding position variables.

$$
\mu_{f_{k} \rightarrow s_{k}}\left(s_{k}\right)=f_{k}\left(s_{k}\right) .
$$

(2) In the second step, we have no message from $\mu_{v_{k} \rightarrow s_{0}}$ yet, so we initialize $\mu_{v_{k} \rightarrow s_{0}}=1, \forall k$. Hence, the messages from $s_{k}$ to $v_{k}$ can be written as:

$$
\begin{aligned}
\mu_{\boldsymbol{s}_{0} \rightarrow v_{k}}\left(\boldsymbol{s}_{0}\right) & =f_{0}\left(\boldsymbol{s}_{0}\right) \prod_{k^{\prime} \neq k}^{L} \mu_{v_{k^{\prime}} \rightarrow s_{0}}\left(\boldsymbol{s}_{0}\right)=f_{0}\left(\boldsymbol{s}_{0}\right), \\
\mu_{\boldsymbol{s}_{k^{\prime}} \rightarrow v_{k^{\prime}}}\left(\boldsymbol{s}_{k^{\prime}}\right) & =f_{k^{\prime}}\left(\boldsymbol{s}_{k^{\prime}}\right) .
\end{aligned}
$$

(3) Next, we compute the outgoing messages from the factor node $v_{k}$ to the variable node $\boldsymbol{a}_{k}$.

$$
\begin{aligned}
& \mu_{v_{0} \rightarrow \boldsymbol{a}_{0}}\left(\boldsymbol{a}_{0}\right)=\int v\left(\boldsymbol{a}_{0}, \boldsymbol{s}_{0}\right) \mu_{\boldsymbol{s}_{0} \rightarrow v_{0}}\left(\boldsymbol{s}_{0}\right) \mathrm{d} \boldsymbol{s}_{0} \\
& \mu_{v_{k^{\prime}} \rightarrow \boldsymbol{a}_{k^{\prime}}}\left(\boldsymbol{a}_{k^{\prime}}\right)=\iint v\left(a_{k^{\prime}}, \boldsymbol{s}_{0}, \boldsymbol{s}_{k^{\prime}}\right) \mu_{\boldsymbol{s}_{k^{\prime}} \rightarrow v_{k^{\prime}}} \mathrm{d} \boldsymbol{s}_{k^{\prime}} d \boldsymbol{s}_{0} .
\end{aligned}
$$

From equation (6c), we can see that $v_{k}$ is already formulated as the product of distributions over all the BSs. Hence, in this step, the messages can be passed in a centralized way, unlike in [13] where the factor graph is formulated in such a way that the messages need to be passed through each BS separately.

(4) Then, we move the message forward to the data association loop. For notational simplicity, we represent the factor node $\psi\left(a_{k}, b_{m}\right)$ by $\psi_{k, m}$. We start by initializing the messages $\mu_{\psi_{k, m} \rightarrow \boldsymbol{a}_{k}}^{(0)}\left(\boldsymbol{a}_{k}\right)=1$. We can then execute multiple iterations. The message in the $p$-th iteration can be written as [14]:

$$
\begin{aligned}
\mu_{\boldsymbol{a}_{k} \rightarrow \psi_{k, \boldsymbol{m}}}^{(p)}\left(\boldsymbol{a}_{k}\right) & =\mu_{v_{k} \rightarrow \boldsymbol{a}_{k}}\left(\boldsymbol{a}_{k}\right) \prod_{\boldsymbol{m}^{\prime} \neq \boldsymbol{m}} \mu_{\psi_{\boldsymbol{m}^{\prime}, k} \rightarrow \boldsymbol{a}_{k}}^{(p-1)}\left(\boldsymbol{a}_{k}\right), \\
\mu_{\psi_{k, \boldsymbol{m}} \rightarrow b_{\boldsymbol{m}}}^{(p)}\left(b_{\boldsymbol{m}}\right) & =\sum_{\boldsymbol{a}_{k}} \psi_{k, \boldsymbol{m}} \mu_{\boldsymbol{a}_{k} \rightarrow \psi_{k, \boldsymbol{m}}}^{(p)}\left(\boldsymbol{a}_{k}\right) \\
\mu_{b_{\boldsymbol{m}} \rightarrow \psi_{k, \boldsymbol{m}}}^{(p)}\left(b_{\boldsymbol{m}}\right) & =\prod_{k^{\prime} \neq k} \mu_{\psi_{k^{\prime}, \boldsymbol{m}} \rightarrow b_{\boldsymbol{m}}}^{(p)}\left(b_{\boldsymbol{m}}\right) \\
\mu_{\psi_{k, \boldsymbol{m}} \rightarrow \boldsymbol{a}_{k}}^{(p)}\left(\boldsymbol{a}_{k}\right) & =\sum_{\boldsymbol{b}_{\boldsymbol{m}}} \psi_{k, \boldsymbol{m}} \mu_{b_{\boldsymbol{m}} \rightarrow \psi_{k, \boldsymbol{m}}}^{(p)}\left(b_{\boldsymbol{m}}\right)
\end{aligned}
$$

(5) After the data association loop, we have the message from $\boldsymbol{a}_{k}$ to $v_{k}$ as:

$$
\mu_{\boldsymbol{a}_{k} \rightarrow v_{k}}\left(\boldsymbol{a}_{k}\right)=\prod_{\boldsymbol{m}} \mu_{\psi_{k, \boldsymbol{m}} \rightarrow \boldsymbol{a}_{k}}\left(\boldsymbol{a}_{k}\right) .
$$


The next step is to calculate the messages $\mu_{v_{k} \rightarrow s_{k}}\left(\boldsymbol{s}_{k}\right)$ and $\mu_{v_{k^{\prime}} \rightarrow s_{0}}\left(s_{0}\right)$. We omit argument for space reasons:

$$
\begin{aligned}
& \mu_{v_{0} \rightarrow \boldsymbol{s}_{0}}\left(\boldsymbol{s}_{0}\right)=\sum_{\boldsymbol{a}_{0}} v\left(\boldsymbol{a}_{0}, \boldsymbol{s}_{0}\right) \mu_{\boldsymbol{a}_{0} \rightarrow v_{0}}\left(\boldsymbol{a}_{0}\right), \\
& \mu_{v_{k^{\prime} \rightarrow \boldsymbol{s}_{0}}}\left(\boldsymbol{s}_{0}\right)=\int \sum_{\boldsymbol{a}_{k^{\prime}}} v_{k^{\prime}} \mu_{a_{k^{\prime}} \rightarrow v_{k^{\prime}}} \mu_{s_{k^{\prime}} \rightarrow v_{k^{\prime}}} \mathrm{d} \boldsymbol{s}_{k^{\prime}}, \\
& \mu_{v_{k^{\prime}} \rightarrow \boldsymbol{s}_{k^{\prime}}}\left(\boldsymbol{s}_{k^{\prime}}\right)=\int \sum_{\boldsymbol{a}_{k}} v_{k^{\prime}} \mu_{a_{k^{\prime}} \rightarrow v_{k^{\prime}}} \mu_{\boldsymbol{s}_{0} \rightarrow v_{k}} \mathrm{~d} \boldsymbol{s}_{0},
\end{aligned}
$$

where, $\mu_{\boldsymbol{s}_{0} \rightarrow v_{k}}\left(\boldsymbol{s}_{0}\right)=\int \sum_{\boldsymbol{a}_{0}} v\left(\boldsymbol{a}_{0}, \boldsymbol{s}_{0}\right) \mu_{a_{0} \rightarrow v_{0}}\left(\boldsymbol{a}_{0}\right) d \boldsymbol{s}_{k^{\prime}}$. (6) Finally, the posterior beliefs can be computed as:

$$
\begin{aligned}
p\left(\boldsymbol{s}_{0} \mid \boldsymbol{Z}\right) \propto \mu_{f_{0} \rightarrow s_{0}}\left(\boldsymbol{s}_{0}\right) \prod_{k} \mu_{v_{k} \rightarrow s_{0}}\left(\boldsymbol{s}_{0}\right) \\
p\left(\boldsymbol{s}_{k^{\prime}} \mid \boldsymbol{Z}\right) \propto \mu_{f_{k^{\prime}} \rightarrow \boldsymbol{s}_{k^{\prime}}}\left(\boldsymbol{s}_{k^{\prime}}\right) \mu_{v_{k^{\prime}} \rightarrow \boldsymbol{s}_{k^{\prime}}}\left(\boldsymbol{s}_{k^{\prime}}\right) .
\end{aligned}
$$

\section{Particle Implementation}

As the integrals in the previous section cannot be solved in closed form, we approximate them using Monte Carlo integration. Thereby we represent the messages as lists of weighted particles. We have to consider that the number of particles are enough to provide an accurate representation of the probability distributions and the weights are normalized such that their sum is 1 . To compute the products in (15a)(15b), we evaluate all messages only in the particles generated from the priors.

\section{Centralized vs Distributed Approach}

For benchmark purposes, we compare our proposed method against a distributed BS based SLAM method equivalent to that in $[13]^{6}$. For the distributed method, we introduce the set $\mathcal{M}_{i}=\left\{1,2, \cdots, \hat{L}_{i}\right\}, \forall i$ for each $i$-th BS. We define $a_{k, i} \triangleq m_{i} \in \mathcal{M}_{i}$ indicating which measurement $m_{i}$ corresponds to the source $k$ at the $i$-th BS. Similarly, we define $b_{m_{i}, i} \triangleq k \in\{0,1, \cdots, L\}$ defining which source corresponds to the measurement $m_{i}$ at the $i$-th BS. Note that contrarily to $\boldsymbol{a}_{k}, b_{\boldsymbol{m}}$ and $\boldsymbol{m}$ in our proposed centralized methods, in this distributed method, $a_{k, i}, b_{m_{i}, i}$ and $m_{i}$ are all scalars and the relation between $\boldsymbol{a}_{i}$ and $b_{i}$, the vector of data association variables, is in fact bijective. We further define

$$
\Psi(\boldsymbol{a}, \boldsymbol{b})=\prod_{i=1}^{N} \prod_{k=0}^{\hat{L}_{i}} \prod_{m_{i} \in \mathcal{M}_{i}} \psi\left(a_{k, i}, b_{m_{i}, i}\right) .
$$

where,

$$
\begin{aligned}
& \psi\left(a_{l, i}, b_{m_{i}, i}\right)= \\
& \begin{cases}0 & \boldsymbol{a}_{k, i}=m_{i}, b_{m_{i}, i} \neq k \text { or } a_{k, i} \neq m_{i}, b_{m_{i}, i}=k \\
1 & \text { otherwise. }\end{cases}
\end{aligned}
$$

Hence, following a formulation similar to the posterior distribution as (4), we can build a factor graph as illustrated in Fig. 3. We can compute the beliefs and accordingly the marginal similarly to the the previous section.

\footnotetext{
${ }^{6}$ In the literature, it is common to consider such distributed methods especially for the data association problem [14], [20]
}

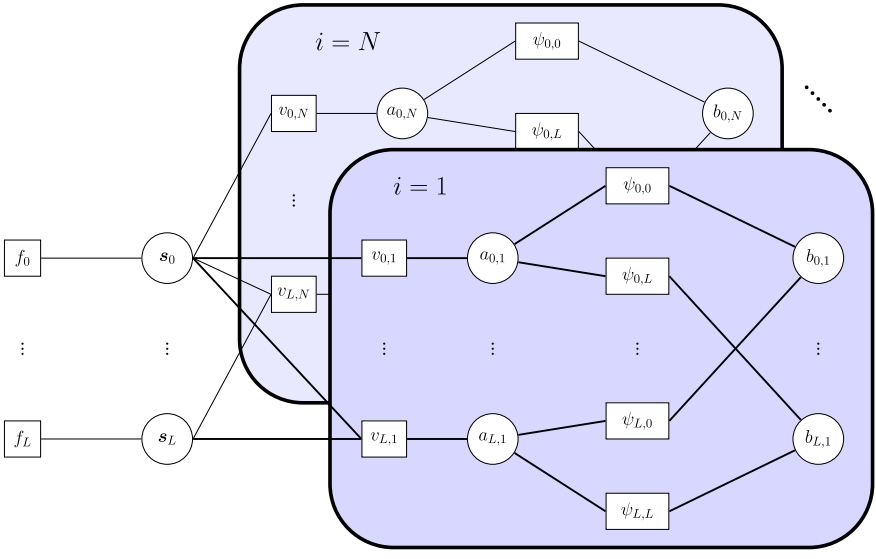

Fig. 3. Factor graph with distributed BP for performance comparison. For notational convenience, $v_{k, i}$ represents $v\left(a_{k, i}, \boldsymbol{s}_{0}, \boldsymbol{s}_{k}\right)$ and $\psi_{k_{1}, k_{2}}=$ $\psi\left(a_{k_{1}, i}, b_{k_{2}, i}\right) \forall i$.

\section{NumERICAL RESUlTS}

In this section, we describe the simulation setup and parameters and then show the simulation of our proposed method. To benchmark our result, we then compare with a model inspired by the distributed BP approach in [13].

\section{A. System parameters and simulation setup}

We consider a scenario with $N=3$ BSs, $L=2$ scatterers and a user where all the BSs can measure all three paths (i.e. $\left.\hat{L}_{i}=3, \forall i\right)$. We consider the locations of BSs are known with $\boldsymbol{x}_{1}=[0,0], \boldsymbol{x}_{2}=[0,100], \boldsymbol{x}_{3}=[100,100]$, whereas the user and the scatters are uniformly distributed within an area of $100 \mathrm{~m} \times 100 \mathrm{~m}$ area.

We assume that the measurement covariance matrix is a diagonal matrix with the variance of estimating AoD $\sigma_{\theta}^{2}$ equal to the variance of estimating the AoA $\sigma_{\phi}^{2}$ for all the measurements. Similarly, while simulating the BP algorithm, we consider $N_{s_{0}}=N_{s_{k^{\prime}}}=2500$ particles (50 per dimension) to represent the distributions of the user and the scatterers.

In our simulations, we assume no prior knowledge of the positions of either the user or the scatterers and hence, we consider they are uniformly distributed within the deployment area. Hence, to replicate $p\left(s_{0}\right)$ as particles, we can draw $N_{s_{0}}$ sample points of $s_{0}$ from the specified domain and assign the corresponding probability as weights, in this case a constant (and accordingly for $p\left(s_{k^{\prime}}\right)$ ).

\section{B. Numerical results and discussion}

In Figs. 4 and 5, we plot the marginal distributions of the user and the scatterers with blue and orange scatter points, as formulated in equations (15a) and (15b) respectively. Since, as said earlier, we are dealing with particles rather than the continuous distribution, the size of blue and orange circles represents the weights associated with the corresponding particles. We also plot the true positions of the BSs, the scatterers and the user for comparison. Between the two figures, we can clearly see that the particle clouds in our proposed method can separate the two scatterers and the user unlike the distributed 


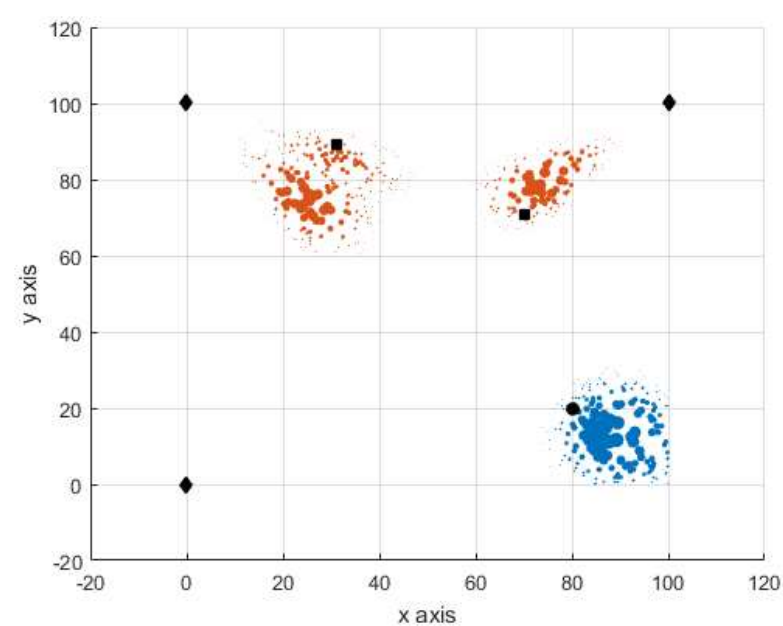

Fig. 4. Marginal distribution with $\sigma_{\theta}^{2}=\sigma_{\phi}^{2}=1 \mathrm{deg}^{2}$ with the proposed method. The diamonds, squares and circle represent the true positions of the 3 BSs, 2 scatterers and the user respectively.

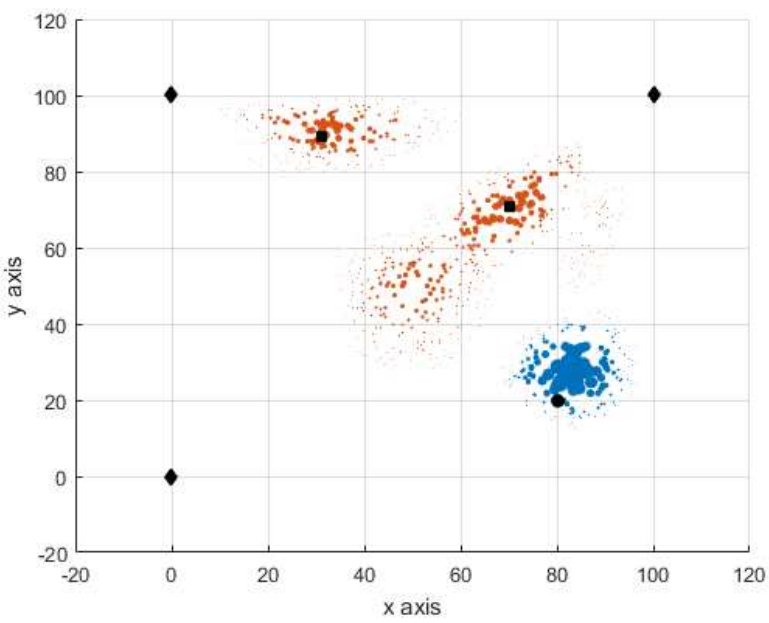

Fig. 5. Marginal distribution with $\sigma_{\theta}^{2}=\sigma_{\phi}^{2}=1 \mathrm{deg}^{2}$ with the distributed method [13]. The diamonds, squares and circle represent the true positions of the $3 \mathrm{BSs}, 2$ scatterers and the user respectively.

$\mathrm{BP}$. The reason is that in our method, as we can deduce from equation (6b), only the particles with non zero probabilities according to equation (2) with respect to all the BSs are passed on from the factor $\boldsymbol{v}$ to the variable $\boldsymbol{a}$ in the factor graph. Hence, in this step, before the data association loop, we do some sort of initial filtering with respect to all the BS. On the contrary, in the distributed method, the filtering is done with respect to only one single BS before the data association loop, and then further filtering is done later during the collection while passing the message from $v_{k}$ to $s_{k}$. Filtering before the loop reduces the propagation of any error during the data association loop.

Following this discussion, in Fig. 6, we plot the empirical cumulative distribution function (CDF) of the root mean squared error (RMSE) between the true and estimated posi-

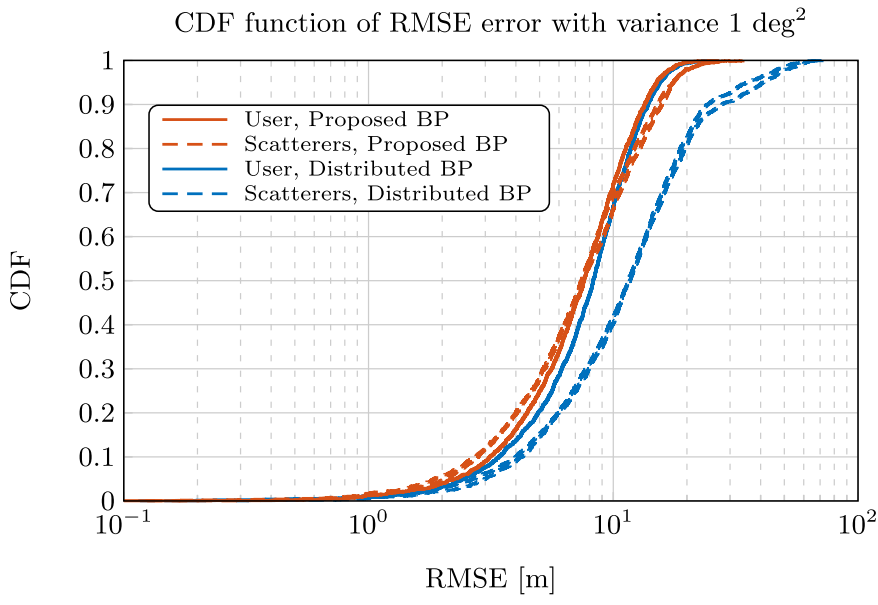

Fig. 6. CDF plot comparison of the RMSE error between the proposed centralized and the distributed BP based methods with $1 \mathrm{deg}^{2}$ variance.

tions of the user and scatterers. We calculate the RMSE by taking the distance between the true and the mean particle position. As we can see, the proposed centralized method performs better than the distributed method especially when it comes to estimating the position of the scatterer due to the limited error propagation in the data association phase.

Comparing the communication overhead between the two approaches, the main difference lies in the fact that in the distributed method, the message between the variable node $s_{k}$ and the factor node $v_{k, i}$ involves exchanging clouds of particles representing the distribution of all the scatterers to and from all the BSs. The overhead cost of such message communication is very high. In contrast, in the proposed centralized method, the only message exchange from the different BSs include the raw AoD and AoA measurement pairs to the centralized unit which is much smaller than the previous.

Regarding the complexity analysis between the two approaches, in the distributed method, we pass $\mathcal{O}\left(N L^{2}\right)$ messages, while in the proposed centralized approach $\mathcal{O}\left(L^{N+1}\right)$ messages. Hence, the centralized approach has better performance and low communication overhead, but at a higher complexity cost. The distributed method exhibits less complexity, but requires exchange of particle clouds among BSs.

\section{CONCLUSIONS}

In this paper, we proposed a BP based method to solve the problem of mm-Wave SLAM by using only angular measurements in a system with multiple BSs and scatterers and a user node. To reach this objective, we firstly formulated the corresponding posterior distribution and then the factor graph including the problem of data association. By running the message passing algorithm in the graph, numerical results demonstrated that even without any a-priori distribution on the positions of the user and the scatterers, one can achieve relatively high accuracy. In comparison with methods from the literature where the data association problem is solved 
in a distributed manner separately at different BSs, we show that our centralized data association approach provides a better estimation accuracy, mostly in terms of scatterers positioning. As a future work, we would like to consider a model including uncertainty in the user orientation and false alarms, which would provide an even more realistic assessment of the problem.

\section{ACKNOWLEDGMENT}

This work was supported, in part, by the European H2020 project SECREDAS, which is funded through the specific ECSEL Joint Undertaking research and innovation program (GA No. 783119), and by the Swedish Research Council under grant No. 2018-03701.

\section{REFERENCES}

[1] T. S. Rappaport, S. Sun, R. Mayzus, H. Zhao, Y. Azar, K. Wang, G. N Wong, J. K. Schulz, M. Samimi, and F. Gutierrez, "Millimeter wave mobile communications for $5 \mathrm{G}$ cellular: It will work!" IEEE Access, vol. 1, pp. 335-349, 2013.

[2] F. Al-Ogaili and R. M. Shubair, "Millimeter-wave mobile communications for 5G: Challenges and opportunities," in 2016 IEEE International Symposium on Antennas and Propagation (APSURSI), Jun 2016, pp. 1003-1004.

[3] Y. Niu, Y. Li, D. Jin, L. Su, and A. V. Vasilakos, "A survey of millimeter wave communications (mmwave) for 5G: opportunities and challenges," Wireless Networks, vol. 21, no. 8, pp. 2657-2676, Nov. 2015. [Online]. Available: https://doi.org/10.1007/s11276-015-0942-z

[4] S. Sun, T. S. Rappaport, R. W. Heath, A. Nix, and S. Rangan, "MIMO for millimeter-wave wireless communications: beamforming, spatial multiplexing, or both?" IEEE Communications Magazine, vol. 52, no. 12 , pp. 110-121, Dec 2014.

[5] S. Bartoletti, A. Conti, D. Dardari, and A. Giorgetti, "5G localization and context-awareness," https://www.5gitaly.eu/wpcontent/uploads/2019/01/5G-Italy-White-eBook-5G-Localization.pdf.

[6] Z. Abu-Shaban, X. Zhou, T. Abhayapala, G. Seco-Granados, and H. Wymeersch, "Error bounds for uplink and downlink 3D localization in 5G millimeter wave systems," IEEE Transactions on Wireless Communications, vol. 17, no. 8, pp. 4939-4954, Aug 2018.

[7] R. Mendrzik, H. Wymeersch, G. Bauch, and Z. Abu-Shaban, "Harnessing NLOS components for position and orientation estimation in 5G millimeter wave mimo," IEEE Transactions on Wireless Communications, vol. 18, no. 1, pp. 93-107, Jan 2019.

[8] A. Shahmansoori, G. E. Garcia, G. Destino, G. Seco-Granados, and $\mathrm{H}$. Wymeersch, "Position and orientation estimation through millimeterwave MIMO in 5G systems," IEEE Transactions on Wireless Communications, vol. 17, no. 3, pp. 1822-1835, Mar 2018.

[9] A. Shahmansoori, B. Uguen, G. Destino, G. Seco-Granados, and $\mathrm{H}$. Wymeersch, "Tracking position and orientation through millimeter wave lens MIMO in 5G systems," CoRR, vol. abs/1809.06343, 2018. [Online]. Available: http://arxiv.org/abs/1809.06343

[10] M. Ulmschneider, R. Raulefs, C. Gentner, and M. Walter, "Multipath assisted positioning in vehicular applications," in 2016 13th Workshop on Positioning, Navigation and Communications (WPNC), Oct 2016, pp. $1-6$.

[11] C. Gentner, R. Pohlmann, M. Ulmschneider, T. Jost, and S. Zhang, "Positioning using terrestrial multipath signals and inertial sensors," Mobile Information Systems, vol. 2017, 2017.

[12] R. Mendrzik, H. Wymeersch, and G. Bauch, "Joint localization and mapping through millimeter wave MIMO in 5G systems," in 2018 IEEE Global Communications Conference (GLOBECOM), Dec 2018, pp. 1-6.

[13] M. Frohle, C. Lindberg, and H. Wymeersch, "Cooperative localization of vehicles without inter-vehicle measurements," in 2018 IEEE Wireless Communications and Networking Conference (WCNC), April 2018, pp. $1-6$.

[14] J. L. Williams and R. A. Lau, "Convergence of loopy belief propagation for data association," in 2010 Sixth International Conference on Intelligent Sensors, Sensor Networks and Information Processing, Dec 2010, pp. $175-180$.
[15] M. Koivisto, M. Costa, J. Werner, K. Heiska, J. Talvitie, K. Leppänen, V. Koivunen, and M. Valkama, "Joint device positioning and clock synchronization in $5 \mathrm{~g}$ ultra-dense networks," IEEE Transactions on Wireless Communications, vol. 16, no. 5, pp. 2866-2881, May 2017.

[16] Y. Wu, B. Peng, H. Wymeersch, G. Seco-Granados, A. Kakkavas, M. H. C. Garcia, and R. A. Stirling-Gallacher, "Cooperative localization with angular measurements and posterior linearization," arXiv preprint arXiv:1907.04700, 2019.

[17] S. Wielandt and L. Strycker, "Indoor multipath assisted angle of arrival localization," Sensors, vol. 17, no. 11, p. 2522, 2017.

[18] F. R. Kschischang, B. J. Frey, H.-A. Loeliger et al., "Factor graphs and the sum-product algorithm," IEEE Transactions on information theory, vol. 47, no. 2, pp. 498-519, 2001.

[19] A. Ihler, J. III, and A. Willsky, "Loopy belief propagation: Convergence and effects of message errors." Journal of Machine Learning Research, vol. 6, pp. 905-936, 052005.

[20] F. Meyer, T. Kropfreiter, J. L. Williams, R. Lau, F. Hlawatsch, P. Braca, and M. Z. Win, "Message passing algorithms for scalable multitarget tracking," Proceedings of the IEEE, vol. 106, no. 2, pp. 221-259, Feb 2018. 